\title{
ASYMPTOTIC SOLUTIONS OF NONLINEAR WAVE EQUATIONS USING THE METHODS OF AVERAGING AND TWO-TIMING*
}

\author{
BY \\ R. W. LARDNER
}

Simon Fraser University, Burnaby, British Columbia

\begin{abstract}
The method of averaging and the two-time procedure are outlined for a class of hyperbolic second-order partial differential equations with small nonlinearities. It is shown that they both lead to the same integro-differential equation for the lowest-order approximation to the solution. For the special case of the nonlinear wave equation, this lowest-order solution consists of the superposition of two modulated travelling waves, and separate integro-differential equations are derived for the amplitudes of these two waves. As an example, the wave equation with Van der Pol type of nonlinearity is considered.
\end{abstract}

1. Introduction. The averaging method, as also its variant, the $\mathrm{KBM}$ method, was developed originally by Krylov and Bogoliubov [1] and Bogoliubov and Mitropolskii [2] for the purpose of providing approximate solutions to ordinary differential equations with small nonlinearities, especially as related to problems of nonlinear vibration. More recently, these methods have been applied to nonlinear partial differential equations in several contexts, among which one can mention the work of Benney [3] and McGoldrick [4] on water wave interactions, of Montgomery and Tidman [5] on plasma waves and of Montgomery [6] on a nonlinear Klein-Gordon equation, the book by Mitropolskii and Moseenkov [7] and the work of Bojadziev and Lardner [8, 9] on waves in nonlinear solids. The present author $[10,11]$ has used the method of averaging to investigate a problem in solid vibrations in which the system possesses infinitely many internal resonances, and has shown that in this case the solution of an arbitrary initial-value problem develops shock layers (i.e., regions in which the solution undergoes rapid changes).

The two-time method was first proposed by Cole and Kevorkian [12], and it was shown by Morrison [13] that for certain classes of ordinary differential equations this method yields solutions identical to those obtained by the method of averaging. This result is significant because of the rigorous foundation of the averaging method by means of a theorem of Bogoliubov's ([2], Chapter VI), which therefore indirectly provides a justification of the two-time method. The two-time method is a special case of the many-variable expansion method [14], which must be used in general when greater accuracy than the lowest-order approximation is required.

Keller and Kogelman [15] have used the two-variable technique to investigate a certain nonlinear partial differential equation (Klein-Gordon equation with a Van der Pol type of nonlinearity) and a similar method has been used by Davy and Ames [16] for a problem concerning the vibrations of a nonlinear viscoelastic rod. In both of these papers, the solution function is expanded in terms of the spatial eigenfunctions of the linearized

* Received February 25, 1976; revised version received September 19, 1976. 
equation and the partial differential equation replaced effectively by an infinite system of ordinary differential equations. As remarked by Keller and Kogelman, it is not hard to see that the two-time method as used in these papers is completely equivalent to the KBM method.

It has been pointed out by Nayfeh [17] that a direct use of the two-time method, without expansion in terms of spatial eigenfunctions, can in certain cases enable the solution of a partial differential equation to be obtained more straightforwardly than through any of the above-mentioned methods. The direct use of a two-time method for certain nonlinear wave equations was first proposed by Chikwendu and Kevorkian [18] and a rigorous basis for some of these authors' results has recently been given by Fink, Hall and Hausrath [19] and by Eckhaus [20]. The purpose of the present paper is to investigate Chikwendu and Kevorkian's and Nayfeh's method in the context of a wide class of hyperbolic partial differential equations, and in particular to compare it with the method of averaging. We shall show that in the lowest approximation, the two methods are formally equivalent for the whole class of equations considered and that for a certain subclass of nonlinear wave equations, the direct two-time method offers considerable computational advantage. This advantage in practical applicability appears, however, to be limited to the simplest differential equations and the simplest boundary conditions. In more complex situations it seems necessary in practice to return to the eigenfunction expansion method and the resulting infinite system of ordinary differential equations.

We shall consider a partial differential equation for the function $u(x, t)$ of the following type:

$$
\rho(x) u_{t t}-\left[k(x) u_{x}\right]_{x}+q(x) u=\epsilon E\left(x, t, u, u_{x}, u_{t}, \cdots, \epsilon\right) .
$$

Here subscripts of $x$ and $t$ denote the corresponding partial derivatives, $\rho(x)>0, k(x)>0$ and $q(x)$ are given functions of $x, \epsilon$ is a small parameter and $E$ a general, but suitably differentiable, function of the indicated arguments. The dots indicate that $E$ may depend on higher-order derivatives of $u$ than those shown explicitly, except that derivatives of order no higher than the second should occur. Eq. (1.1) is assumed to hold for $t>0$, with the associated initial conditions that $u(x, 0)$ and $u_{t}(x, 0)$ are prescribed. Throughout most of the paper it will be supposed that the variable $x$ is restricted to the interval $(0, l)$ with $u$ satisfying a pair of linear boundary conditions involving the values of $u$ and $u_{x}$ at the two endpoints $x=0$ and $x=l$. We shall write these boundary conditions in the symbolic form $\Re_{1}\{u\}=\Re_{2}\{u\}=0$, where

$$
B_{i}\{u\}=A_{i} u(0, t)+B_{i} u_{x}(0, t)+C_{i} u(l, t)+D_{i} u_{x}(l, t) \quad(i=1,2)
$$

for certain given values of the eight constants $\left\{A_{1}, \ldots, D_{2}\right\}$.

In Sec. 2, the approximate solution of Eq. (1.1) by means of the method of averaging is outlined. In general, this method of solution leads to a coupled infinite system of ordinary differential equations for the amplitudes of the different modes in the lowest-order solution. However, it is shown that this system is equivalent to a single integro-differential equation for the lowest-order solution itself. In Sec. 3, the two-time method is described and is shown to lead to the same integro-differential equation, thus establishing the equivalence of the two methods as far as the lowest-order approximation is concerned.

In Sec. 4 of the paper, the particular case of the nonlinear wave equation is considered corresponding to the choice $\rho(x)=k(x)=1, q(x)=0$ in the general equation (1.1). For this case, the lowest-order solution can be represented as the superposition of two modulated waves, one travelling in the positive, the other in the negative $x$-direction, and separate integro-differential equations are found for the amplitudes of these two waves. 
With a general nonlinear function $E$ in the original wave equation, these two amplitudes are coupled, although a particular example is given in which they become uncoupled.

The practical usefulness of this representation of the solution in terms of two waves depends on the complexity of the two boundary conditions. For the special case of fixedend conditions, the technique of odd extension enables the method to be used successfully, and the method becomes especially convenient when the nonlinear function $E$ satisfies a pair of invariance requirements. In a similar way, for boundary conditions of free end type, the analogous technique of even extension enables the solution to be found, with $E$ satisfying a (different) pair of invariance conditions. For more complex boundary conditions, it appears to be necessary in practice to return to the eigenfunction expansion as a method of solution.

As an example, the wave equation with Van der Pol type of nonlinearity, namely

$$
u_{t t}-u_{x x} \epsilon\left(u_{t}-\frac{1}{3} u_{t}^{3}\right)
$$

is considered in the case of fixed-end conditions, $u(0, t)=u(l, t)=0$. This equation has been used by Myerscough [21] to investigate the wind-induced oscillations of overhead powerlines, and the analytical results obtained in the present paper shed further light on that problem.

The wave equation with van der Pol type of nonlinearity has also been investigated by Chikwendu and Kevorkian [18] for a particular case of initial data. In the present paper, arbitrary initial conditions of impulsive type are allowed, and the general asymptotic form of the solution as $t \rightarrow \infty$ is found explicitly.

\section{The averaging method.}

2.1 The linearized equation. Consider first the equation obtained by setting $\epsilon=0$ in Eq. (1.1),

$$
\rho(x) u_{t t}-\left[k(x) u_{x}\right]_{x}+q(x) u=0,
$$

with $u$ again required to satisfy the boundary conditions $B_{t}\{u\}=0(i=1,2)$. This equation possesses separable solutions of the form

$$
u=a_{n} \phi_{n}(x) \cos \left(\omega_{n} t+\alpha_{n}\right)
$$

where $a_{n}$ and $\alpha_{n}$ are arbitrary constants and $\phi_{n}(x)$ satisfies

$$
\left[k(x) \phi_{n}{ }^{\prime}(x)\right]^{\prime}+\left[\rho(x) \omega_{n}{ }^{2}-q(x)\right] \phi_{n}(x)=0, \quad B_{i}\left\{\phi_{n}\right\}=0 \quad(i=1,2) .
$$

There is an infinite system of such solutions and, provided the two boundary conditions satisfy the well-known self-adjointness condition of Sturm-Liouville theory, the set of eigenfunctions $\left\{\phi_{n}(x)\right\}$ forms a complete set, orthogonal with respect to the weight function $\rho(x)$. By appropriate normalization of the eigenfunctions therefore we have the result that

$$
\int_{0}^{l} \rho(x) \phi_{m}(x) \phi_{n}(x) d x=\delta_{m n},
$$

where $\delta_{m n}$ is the Kronecker symbol.

The general solution of Eq. (2.1) may be taken in the form of a sum of separable solutions,

$$
u(x, t)=\sum_{n=1}^{\infty} a_{n} \phi_{n}(x) \cos \left(\omega_{n} t+\alpha_{n}\right) .
$$


The index $n$ is chosen to run over the positive integers, and it is assumed that all of the eigenfrequencies $\left\{\omega_{n}\right\}$ which are admitted by the boundary conditions are non-zero. The coefficients $\left\{a_{n}, \alpha_{n}\right\}$ appearing in the expansion (2.4) are usually determined in terms of the initial values of $u$ and $u_{t}$.

By expanding the cosine and defining $z_{n}=a_{n} \exp \left(i \alpha_{n}\right)(n=1,2, \ldots)$, the solution (2.4) may be rewritten as

$$
u(x, t)=\frac{1}{2} \sum_{n=1}^{\infty}\left(z_{n} \exp \left(i \omega_{n} t\right)+\bar{z}_{n} \exp \left(-i \omega_{n} t\right)\right) \phi_{n}(x)
$$

The corresponding expressions for the first derivatives of $u$ are

$$
\begin{aligned}
& u_{x}(x, t)=\frac{1}{2} \sum_{n=1}^{\infty}\left(z_{n} \exp \left(i \omega_{n} t\right)+z_{n} \exp \left(-i \omega_{n} t\right)\right) \phi_{n}{ }^{\prime}(x), \\
& u_{t}(x, t)=\frac{1}{2} \sum_{n=1}^{\infty} i \omega_{n}\left(z_{n} \exp \left(i \omega_{n} t\right)-z_{n} \exp \left(-i \omega_{n} t\right)\right) \phi_{n}(x) .
\end{aligned}
$$

2.2 The nonlinear equation. In order to use the averaging method to solve the nonlinear equation (1.1), we continue to assume that the solution has the form given by Eqs. (2.5)-(2.7), but with the coefficients $\left\{z_{n}\right\}$ now being slowly varying functions of time instead of constants. This will be expressly indicated by writing $z_{n}=z_{n}(\tau)$ as functions of the "slow" time variable $\tau=\epsilon t$. Then the solution is sought in the form

$$
\begin{gathered}
u(x, t)=F(x, t, \tau)=\frac{1}{2} \sum_{n=1}^{\infty}\left[z_{n}(\tau) \exp \left(i \omega_{n} t\right)+\overline{z_{n}(\tau)} \exp \left(-i \omega_{n} t\right)\right] \phi_{n}(x) \\
u_{x}(x, t)=F_{x}(x, t, \tau)=\frac{1}{2} \sum_{n=1}^{\infty}\left[z_{n}(\tau) \exp \left(i \omega_{n} t\right)+\overline{z_{n}(\tau)} \exp \left(-i \omega_{n} t\right)\right] \phi_{n}{ }^{\prime}(x) \\
u_{t}(x, t)=F_{t}(x, t, \tau)=\frac{1}{2} \sum_{n=1}^{\infty} i \omega_{n}\left[z_{n}(\tau) \exp \left(i \omega_{n} t\right)-\overline{z_{n}(\tau)} \exp \left(-i \omega_{n} t\right)\right] \phi_{n}(x)
\end{gathered}
$$

Eqs. (2.8) and (2.10) are compatible only if $z_{n}(\tau)$ satisfies the condition that

$$
z_{n}^{\prime} \exp \left(i \omega_{n} t\right)+\overline{z_{n}^{\prime}} \exp \left(-i \omega_{n} t\right)=0
$$

where $z_{n}{ }^{\prime}$ is used to denote $d z_{n} / d \tau$.

Substituting Eqs. (2.8)-(2.10) into the nonlinear differential equation (1.1) and making use of Eq. (2.2), we arrive at the result that

$$
\frac{1}{2} \sum_{n=1}^{\infty} i \omega_{n}\left[z_{n}{ }^{\prime} \exp \left(i \omega_{n} t\right)-\overline{z_{n}{ }^{\prime}} \exp \left(-i \omega_{n} t\right)\right] \rho(x) \phi_{n}(x)=E(x, t,\{F(x, t, \tau)\}, \epsilon)(2.12)
$$

where $E(x, t,\{F(x, t, \tau)\}, \epsilon)$ is used as an abbreviation for the function $E$ appearing on the right-hand side of Eq. (1.1) when Eqs. (2.8)-(2.10) have been substituted for $u$ and its first derivatives and corresponding expressions for the higher derivatives of $u$. Multiplying through Eq. (2.12) by $\phi_{n}(x)$, integrating over $0 \leq x \leq l$ and making use of the orthonormality condition $(2.3)$, we obtain that

$$
\frac{1}{2} i \omega_{n}\left[z_{n}{ }^{\prime} \exp \left(i \omega_{n} t\right)-\overline{z_{n}{ }^{\prime}} \exp \left(-i \omega_{n} t\right)\right]=E_{n}\left(t,\left\{z_{l}\right\}, \epsilon\right)
$$


where

$$
E_{n}\left(t,\left\{z_{l}\right\}, \epsilon\right)=\int_{0}^{l} E(x, t,\{F(x, t, \tau)\}, \epsilon) \phi_{n}(x) d x .
$$

Combining Eqs. (2.11) and (2.13) therefore leads to the result that

$$
z_{n}{ }^{\prime}(\tau)=-i \omega_{n}{ }^{-1} E_{n}\left(t,\left\{z_{l}\right\}, \epsilon\right) \exp \left(-i \omega_{n} t\right) .
$$

This is an infinite system of coupled differential equations for the amplitudes $\left\{z_{n}(\tau)\right\}$.

2.3 The averaged system. According to the method of averaging (in lowest order), the system of equations $(2.15)$ is replaced by the autonomous system

$$
z_{n}^{\prime}(\tau)=\lim _{T \rightarrow \infty}-\frac{i}{\omega_{n} T} \int_{0}^{T} E_{n}\left(t,\left\{z_{l}\right\}, 0\right) \exp \left(-i \omega_{n} t\right) d t
$$

where in performing the integration over $t$ the variables $\left\{z_{l}\right\}$ are held fixed. By virtue of a theorem of Bogoliubov's [2,19], if $\tau$ is restricted to some finite interval $0 \leq \tau \leq L$, where $L$ is any constant, the difference between the solutions $z_{n}(\tau)$ of the original system $(2.15)$ and of the averaged system (2.16) is $o(1)$ as $\epsilon \rightarrow 0$. It is of course assumed that the initial values $z_{n}(0)$ of these two solutions are the same. (It should be noted that the proof of this theorem has been given only for finite systems of equations, whereas Eq. (2.15) forms an infinite system. We shall however assume that the theorem applies in this case.)

Within the approximation of the method of averaging, a differential equation may be obtained for $F(x, t, \tau)$ which is equivalent to the approximate equations (2.16). By differentiating Eq. (2.8) with respect to $\tau$ and substituting from Eqs. (2.16) we obtain the result that

$$
F_{\tau}(x, t, \tau)=\sum_{n=1}^{\infty} \lim _{T \rightarrow \infty} \frac{1}{T} \int_{0}^{T} E_{n}\left(s,\left\{z_{l}\right\}, 0\right) \omega_{n}^{-1} \sin \omega_{n}(t-s) \phi_{n}(x) d s .
$$

After substituting from Eq. (2.14) we find that

$$
F_{\tau}(x, t, \tau)=\lim _{T \rightarrow \infty} \frac{1}{T} \int_{0}^{T} \int_{0}^{l} K(x, y, t-s) E(y, s,\{F(y, s, \tau)\}, 0) d y d s
$$

where

$$
K(x, y, t-s)=\sum_{n=1}^{\infty} \omega_{n}^{-1} \phi_{n}(x) \phi_{n}(y) \sin \omega_{n}(t-s) .
$$

The function $K$ defined by Eq. (2.18) is, for $t-s>0$, equal to the Green's function for the partial differential operator appearing on the left of Eq. (1.1). If $v(x, t)$ satisfies the system of equations

$$
\begin{gathered}
\rho(x) v_{t t}-\left[k(x) v_{x}\right]_{x}+q(x) v=f(x, t) \\
\oiint_{i}\{v\}=0 \quad(i=1,2), \quad v(x, 0)=v_{t}(x, 0)=0,
\end{gathered}
$$

then by use of the Green's function $K$, one can express $v(x, t)$ in terms of the function $f$ appearing on the right of Eq. (2.19):

$$
v(x, t)=\int_{0}^{t} \int_{0}^{l} K(x, y, t-s) F(y, s) d y d s .
$$

It should be noted, however, that according to its definition $(2.18) K(x, y, t-s)$ is an 
odd function of $(t-s)$, and does not vanish for the values $s>t$ which appear in the integral in Eq. (2.17).

Assuming that the Green's function $K$ is known, Eq. (2.17) provides an integrodifferential equation for the function $F$ in terms of which the solution for $u$ and its derivatives is given by Eqs. (2.8)-(2.10). On the basis of our hypothesis that Bogoliubov's theorem extends to the present case, this equation provides an approximation to the solution of Eq. (1.1) which for $0 \leq t \leq L \epsilon^{-1}$ is within an error $o$ (1) of the exact solution.

Past uses of the method of averaging or the $K B M$ method for partial differential equations have involved the direct use of the infinite system of equations (2.16) [3-10] (cf. also $[15,16])$. The integro-differential equation (2.17) has considerable computational advantage in some cases over these earlier approaches. This will become apparent in Sec. 4 when we shall examine in closer detail the form of Eq. (2.17) for the wave equation in which $\rho(x)=k(x)=1$ and $q(x)=0$.

3. The two-time method. We shall now consider the solution of Eq. (1.1) by means of the two-time expansion method. According to this method the solution is sought in the form $[17,18]$

$$
u(x, t)=u_{0}(x, t, \tau)+\epsilon u_{1}(x, t, \tau)+0\left(\epsilon^{2}\right)
$$

where $\tau=\epsilon t$. This expansion is assumed to be uniform in the sense that the ratio $\epsilon u_{1} / u_{0}$ of the second to the first terms is $o(1)$ over the whole range of $t$ which is of interest (excluding neighborhoods of points where $u_{0}=0$ ). Since we wish to find a solution which is valid for $t$ as large as $0\left(\epsilon^{-1}\right)$, we much therefore require that $u_{1}$ does not contain terms which grow linearly with time-that is, we must impose the condition that

$$
\lim _{t \rightarrow \infty} t^{-1} u_{1}(\dot{x}, t, \tau)=0 \text {. }
$$

Substituting Eq. (3.1) into Eq. (1.1) and comparing the terms of orders $\epsilon^{0}$ and $\epsilon^{1}$, we arrive at the two equations

$$
\begin{gathered}
\rho(x) u_{0 t t}-\left[k(x) u_{0 x}\right]_{x}+q(x) u_{0}=0 \\
\rho(x) u_{1 t t}-\left[k(x) u_{1 x}\right]_{x}+q(x) u_{1}=-2 \rho(x) u_{0 t \tau}+E\left(x, t,\left\{u_{0}(x, t, \tau)\right\}, 0\right) .
\end{gathered}
$$

Eq. (3.3) is the same as the homogeneous equation (2.1) with the difference only that the variable $\tau$ enters as a parameter. Thus the solution $u_{0}(x, t, \tau)$ may be found in the form (2.4) in which the coefficients $\left\{a_{n}, \alpha_{n}\right\}$ are allowed to be functions of $\tau$. Equivalently, therefore, we may write $u_{0}(x, t, \tau)=F(x, t, \tau)$ where $F$ can be expanded in the form given in Eq. (2.8).

By substituting this solution into Eq. (3.4) the following equation for $u_{1}$ is obtained:

$$
\begin{aligned}
\rho(x) u_{1 t t}-\left[k(x) u_{1 x}\right]_{x}+ & q(x) u_{1}= \\
& -2 \rho(x) F_{t \tau}(x, t, \tau)+E(x, t,\{F(x, t, \tau)\}, 0) .
\end{aligned}
$$

The solution can be written down by making use of the Green's function via Eqs. (2.19), (2.20) and (2.21), and takes the form

$$
\begin{aligned}
u_{1}(x, t, \tau)=\int_{0}^{t} \int_{0}^{l} K(x, y, t & -s)\left[-2 \rho(y) F_{s \tau}(y, s, \tau)\right. \\
& +E(y, s,\{F(y, s, \tau)\}, 0)] d y d s+F_{1}(x, t, \tau)
\end{aligned}
$$


in which $F_{1}$ is simply a solution of the homogeneous equation (2.1) which appears as a complementary function. $F_{1}$ must be included in $u_{1}$ since in general the initial conditions satisfied by $u_{1}$ are not homogeneous.

In order to avoid secular terms in $u_{1}$, condition (3.2) is imposed. This condition is met if we require that

$\lim _{T \rightarrow \infty} \frac{1}{T} \int_{0}^{T} \int_{0}^{l} K(x, y, t-s)\left[-2 \rho(y) F_{s \tau}(y, s, \tau)+E(y, s\{F(y, s, \tau)\}, 0)\right] d y d s=0$.

Note that in the integrand in Eq. (3.6) the variable $t$ has been kept as a free variable rather than set equal to $T$, so that Eq. (3.6) is a stronger condition than (3.2). Provided that the limit in Eq. (3.6) converges uniformly in $t$ for sufficiently large $t$, then Eq. (3.6) implies that condition (3.2) holds.

If, in the first term in the above integral, the values of $K$ and $F$ are substituted from Eqs. (2.8) and (2.18) and the integration over $y$ performed by use of Eq. (2.3), we arrive at the expression

$$
-\lim _{T \rightarrow \infty} \frac{1}{T} \int_{0}^{T} \sum_{n=1}^{\infty} i\left[z_{n}{ }^{\prime}(\tau) \exp \left(i \omega_{n} s\right)-\overline{z_{n}{ }^{\prime}}(\tau) \exp \left(i \omega_{n} s\right)\right] \sin \omega_{n}(t-s) d s \phi_{n}(x)
$$

for this term. This may readily be evaluated and turns out to be $-F_{\tau}(x, t, \tau)$. Thus the condition (3.6) leads to the same integro-differential equation for $F$, namely Eq. (2.17), as that obtained from the method of averaging. In this way, it is seen that the two methods lead to identical approximate solutions, at least in the lowest order of approximation.

In the particular case of the nonlinear wave equation $(\rho=k=1, q=0)$ with sufficiently simple boundary conditions it is possible to write down directly the solution of Eq. (3.5) without formally introducing a Green's function [17, 18]. The secular terms in $u_{1}$ then become apparent, and equating them to zero leads to the same result as Eq. (2.17). This more traditional use of the two-time approach is somewhat more economical than the use of Eq. (2.17), though is of less general applicability, insofar as (2.17) holds formally for all equations of the type (1.1).

\section{The wave equation with small nonlinearity.}

4.1 Infinite region. In this section we shall consider the special case of Eq. (1.1) in which $\rho(x)=k(x)=1$ and $q(x)=0$, that is, the nonlinear wave equation

$$
u_{t t}-u_{x x}=\epsilon E\left(x, t, u, u_{x}, u_{t}, \cdots, \epsilon\right) .
$$

We shall begin in this first subsection by considering the case when the finite interval $(0, l)$ is replaced by the infinite interval $(-\infty, \infty)$. This may be accomplished in effect by replacing $(0, l)$ by the finite interval $(-L, L)$ and taking $L$ so large that, for values of $x$ near to zero, the boundaries at $\pm L$ have no effect within the interval of time which is of interest. (Alternatively, the sum appearing in Eq. (2.4) can be replaced by an appropriate integral transform to allow an infinite region to be used.)

The function $F(x, t, \tau)$ defined by Eq. (2.8) can be regarded as simply a general solution of the homogeneous equation (2.1) in which $\tau$ appears as a parameter. In the special case of the wave equation, such a solution takes the form of a linear combination of a function of $t+x$ and a function of $t-x$, so that we can write

$$
u_{0}(x, t, \tau)=F(x, t, \tau)=G(t+x, \tau)-H(t-x, \tau)
$$


for certain functions $G$ and $H$. These two functions are not uniquely determined, as an arbitrary function of $\tau$ can be added to each of them without changing $F$.

As is well known, the Green's function for the wave operator in an infinite region can be expressed in closed form and is given by the formula

$$
\begin{aligned}
& =1 / 2 \text { for } x-t<y<x+t, t>0 \\
K(x, y, t) & =-1 / 2 \text { for } x+t<y<x-t, t<0 \\
& =0 \text { otherwise. }
\end{aligned}
$$

Therefore Eq. (2.17) takes the form

$$
\begin{aligned}
G_{\tau}(t+x, \tau)- & H_{\tau}(t-x, \tau) \\
& =\lim _{T \rightarrow \infty} \frac{1}{2 T} \int_{0}^{T} \int_{x-t+s}^{x+t-s} E(y, s,\{G(s+y, \tau)-H(s-y, \tau)\}, 0) d y d s .
\end{aligned}
$$

In this identity we can compare the parts depending on $t+x$ and $t-x$, arriving at the result that

$$
\begin{aligned}
& G_{\tau}(\theta, \tau)=\lim _{\tau \rightarrow \infty} \frac{1}{2 T} \int_{0}^{T} \int_{y_{0}}^{\theta-s} E(y, s,\{G(s+y, \tau)-H(s-y, \tau)\}, 0) d y d s \\
& H_{\tau}(\theta, \tau)=\lim _{\tau \rightarrow \infty} \frac{1}{2 T} \int_{0}^{T} \int_{y_{0}}^{s-\theta} E(y, s,\{G(s+y, \tau)-H(s-y, \tau)\}, 0) d y d s .
\end{aligned}
$$

The lower limit $y_{0}$ of the $y$ integration range can be chosen arbitrarily; a particular choice of this limit as a function of $\tau$ resolves the above-mentioned ambiguities in $G$ and $H$, apart from a constant.

It is generally more convenient to use Eqs. (4.3) in a form obtained by differentiating with respect to $\theta$,

$$
\begin{aligned}
& G_{\theta_{\tau}}(\theta, \tau)=\lim _{T \rightarrow \infty} \frac{1}{2 T} \int_{0}^{T} E(\theta-s, s,\{G(\theta, \tau-H(2 s-\theta, \tau)\}, 0) d s \\
& H_{\theta_{\tau}}(\theta, \tau)=\lim _{T \rightarrow \infty} \frac{-1}{2 T} \int_{0}^{T} E(s-\theta, s,\{G(2 s-\theta, \tau)-H(\theta, \tau)\}, 0) d s .
\end{aligned}
$$

In the particular case when $E$ depends only on $u_{x}$ and $u_{t}$, Eqs. (4.4) have been obtained previously by Chikwendu and Kevorkian [18] and have been given a rigorous basis by Eckhaus [19].

As an illustration of the use of this result, let us consider the partial differential equation

$$
u_{t t}-u_{x x}=\epsilon\left(2 u_{x} u_{x x}+k u_{x x t}\right)
$$

which arises in consideration of the plane vibrations of a material which is predominantly linearly elastic but which also possesses small quadratic elasticity and small linear viscoelasticity [11]. In this case, the first of Eqs. (4.4) becomes

$$
\begin{aligned}
G_{\theta \tau}(\theta, \tau) & =\lim _{T \rightarrow \infty} \frac{1}{2 T} \int_{0}^{T}\left\{2\left[G_{\theta}(\theta, \tau)-H_{\theta}(2 s-\theta, \tau)\right]\left[G_{\theta \theta}(\theta, \tau)-H_{\theta \theta}(\theta, \tau)\right]\right. \\
& \left.+k\left[G_{\theta \theta \theta}(\theta, \tau)+H_{\theta \theta \theta}(2 s-\theta, \tau)\right]\right\} d s
\end{aligned}
$$




$$
\begin{aligned}
& =\lim _{T \rightarrow \infty} \frac{1}{T}\left\{T\left[G_{\theta}(\theta, \tau) G_{\theta \theta}(\theta, \tau)+\frac{1}{2} k G_{\theta \theta \theta}(\theta, \tau)\right]+\frac{1}{2}\left[G_{\theta \theta}(\theta, \tau) H(2 s-\theta, \tau)\right.\right. \\
& \left.\left\llcorner G_{\theta}(\theta, \tau) H_{\theta}(2 s-\theta, \tau)-\frac{1}{2}\left\{H_{\theta}(2 s-\theta, \tau)\right\}^{2}-\frac{1}{2} k H_{\theta \theta}(2 s-\theta, \tau)\right]_{s}^{s=T}=0\right\} \\
& =G_{\theta}(\theta, \tau) G_{\theta \theta}(\theta, \tau)+\frac{1}{2} k G_{\theta \theta \theta}(\theta, \tau) .
\end{aligned}
$$

Similarly the second equation becomes

$$
H_{\theta r}(\theta, \tau)=H_{\theta}(\theta, \tau) H_{\theta \theta}(\theta, \tau)+\frac{1}{2} k H_{\theta \theta \theta}(\theta, \tau) .
$$

By setting $G_{\theta}=g$ and $H_{\theta}=h$, these become Burgers' equation for $g$ and $h$.

In this example, the differential equations (4.6) and (4.7) satisfied by $G$ and $H$ separate: as has been pointed out elsewhere $[22,23,11]$, this particular nonlinearity produces no coupling between the forward-propagating and backward-propagating parts, $G$ and $H$, of the lowest-order solution (4.2). This result would not of course hold true for a general form of nonlinearity in Eq. (4.1). In fact, if one includes cubic elasticity in the above example, then the two parts of the solution (4.2) do interact [24].

Although a general nonlinearity introduces coupling between the forward- and backward-propagating parts of the lowest-order solution, it is of interest to ask whether, for a given nonlinearity, particular solutions exist which involve only one of these components. For example, let us seek a solution of the form $u_{0}(x, t)=G(t+x, \tau)$ involving only waves propagating to the left. Then Eqs. (4.4) take the form

$$
\begin{gathered}
G_{\theta \tau}(\theta, \tau)=\lim _{T \rightarrow \infty} \frac{1}{2 T} \int_{0}^{T} E(\theta-s, s,\{G(\theta, \tau)\}, 0) d s, \\
\lim _{T \rightarrow \infty} \frac{1}{T} \int_{0}^{T} E(s-\theta, s,\{G(2 s-\theta, \tau)\}, 0) d s=0 .
\end{gathered}
$$

For a wave of this type, the initial conditions on $u$ must be of the form

$$
u(x, 0)=p(x), u_{t}(x, 0)=p^{\prime}(x)
$$

for some function $p(x)$. Then $G(\theta, 0)=p(\theta)$, and with these intial values the first of Eqs. $\left(4.4^{\prime}\right)$ can be solved for $G(\theta, \tau)$. The second of these equations then becomes a constraint on the initial data function $p(x)$ which must be satisfied in order that a unidirectional wave type of solution should exist.

Several solutions of this type were found by Chikwendu and Kevorkian in Sec. 3 of their paper for a number of different nonlinearities but for a particular choice of $p(x)$. In this paper it was not stressed that the possibility of existence of unidirectional waves depends on the choice of $p(x)$, although the particular choice of $p(x)$ which was made does meet the required condition.

In the special case when $E(x, t,\{G\}, 0)=E\{G\}$, not depending explicitly on $x$ and $t$, the first of Eqs. (4.4') reduces to the differential equation

$$
G_{\theta \tau}(\theta, \tau)=\frac{1}{2} E\{G(\theta, \tau)\}
$$

In this case the second of Eqs. $\left(4.4^{\prime}\right)$ can be transformed to the simple condition

$$
\lim _{T \rightarrow \infty} \frac{1}{T} G_{\tau}(2 T-\theta, \tau)=0
$$


which must be satisfied by the solution $G$.

4.2 Finite region; fixed end conditions. We now consider the case in which $u(x, t)$ satisfies the nonlinear wave equation (4.1) in the region $0<x<l$, together with boundary conditions at $x=0, l$ of fixed-end type: $u(0, t)=u(l, t)=0$. In this case, the technique of odd extension may be used, just as for the linear wave equation. According to this technique, the function $u(x, t)$ is extended to all values of $x$ in such a way as to be odd about $x=0$ and $x=l$ (i.e., $u(-x, t)=-u(x, t), u(2 l-x, t)=-u(x, t))$. The extended function is automatically periodic in $x$ with period $2 l$.

In order that the extended function $u(x, t)$ should satisfy Eq. (4.1) for all values of $x$, it is necessary that the function $E$ on the right-hand side also be extended. Thus we require that

$$
\begin{aligned}
E\left(-x, t,-u, u_{x},-u_{t}, \cdots, \epsilon\right) & =-E\left(x, t, u, u_{x}, u_{t}, \cdots, \epsilon\right) \\
E\left(x+2 l, t, u, u_{x}, u_{t}, \cdots, \epsilon\right) & =E\left(x, t, u, u_{x}, u_{t}, \cdots, \epsilon\right) .
\end{aligned}
$$

If $E$ is extended in this way, Eq. (4.1) is satisfied for $-\infty<x<\infty$, and the results of the previous subsection can be immediately taken over.

It may happen that the extension of $E$ defined by Eqs. (4.8) leads to a cumbersome form for the right-hand side of the differential equation. For example, in the simple case when $E=u_{t}{ }^{2}(0<x<l)$, the extended $E$ is only defined piecewise, having the value $(-1)^{k} u_{t}{ }^{2}$ when $x$ lies in the interval $k l<x<(k+1) l$. However, in many cases the extended $E$ will be more convenient-for example, in the case of Eq. (4.5), the extended function $E$ is given by $E=2 u_{x} u_{x x}+k u_{x x t}$ for all $x$. In such cases the method of odd extension provides a considerable advantage.

When $u_{0}=F(x, t, \tau)$ is given by Eq. (4.2), the requirement that it be odd about $x=0$ and $x=l$ is met provided that $G(\theta, \tau)=H(\theta, \tau)$ and that $G(\theta, \tau)$ is periodic in $\theta$ with period $2 l$. For consistency, it is necessary that the two equations (4.4) should become identical when $H$ is set equal to $G$ and that each of these equations should be invariant under the transformation $\theta \rightarrow \theta+2 l$. These requirements can be seen to follow from the conditions (4.8) on $E$.

For the case of fixed end conditions, simplified versions of Eqs. (4.4) may be derived. We note that, in view of condition (4.8b) and the periodity of $G$, the integrands in Eqs. (4.4) are periodic in $s$ with period $2 l$ provided that in its explicit time dependence the nonlinear function $E$ is taken to be $2 l$-periodic. With this restriction, the long-time averages in Eqs. (4.4) can be replaced by averages over a single period of length $2 l$ :

$$
G_{\theta \tau}(\theta, \tau)=\frac{1}{4 l} \int_{\theta-l}^{\theta+l} E(\theta-s, s\{G(\theta, \tau)-G(2 s-\theta, \tau)\}, 0) d s .
$$

Dividing the integration range into two halves, from $\theta-l$ to $\theta$ and from $\theta$ to $\theta+l$, making use of Eq. (4.8a) in the lower of these two regions of integration, then introducing the variable $\sigma=2 s-\theta$, we obtain the simpler equation

$$
\begin{aligned}
G_{\theta}(\theta, \tau)=\frac{1}{8 l}\left\{\int _ { \theta } ^ { 2 l + \theta } E \left[\frac{\theta-\sigma}{2}, \frac{\theta+\sigma}{2}\right.\right. & ,\{G(\theta, \tau)-G(\sigma, \tau)\}, 0] d \sigma \\
& \left.-\int_{-2 l+\theta}^{\theta} E\left[\frac{\sigma-\theta}{2}, \frac{\sigma+\theta}{2},\{G(\sigma, \tau)-G(\theta, \tau)\}, 0\right] d \sigma\right\} .
\end{aligned}
$$

In view of the periodity of $G(\sigma, \tau)$, the special case in which $E$ does not depend explicity on $x$ and $t$ corresponds to the integro-differential equation 


$$
G_{\theta \tau}(\theta, \tau)=\frac{1}{8 l} \int_{0}^{2 l}\{E(\{G(\theta, \tau)-G(\sigma, \tau)\}, 0)-E(\{G(\sigma, \tau)-G(\theta, \tau)\}, 0)\} d \sigma .
$$

4.3 Generalized Van der Pol equation. In this section we shall investigate the partial differential equation

$$
u_{t t}-u_{x x}=\epsilon\left(u_{t}-\frac{1}{3} u_{t}^{3}\right), \quad u(0, t)=u(l, t)=0
$$

which is a generalization of the well-known Van der Pol equation.

We note first of all that when the right-hand side is extended according to Eqs. (4.8) it maintains the form $E=u_{t}-\frac{1}{3} u_{t}{ }^{3}$, so that the first of Eqs. (4.9) can be taken to hold for all $x$. The lowest-order solution $u_{0}$ is given by Eq. (4.2) with $G(\theta, \tau)$ and $H(\theta, \tau)$ equal to one another and each periodic with period $2 l$.

The requirement that the second term $u_{1}$ should not contain secular terms leads to Eqs. (4.4) which in the present case take the form of the single equation

$$
G_{\theta \tau}(\theta, \tau)=\lim _{T \rightarrow \infty} \frac{1}{2 T} \int_{0}^{T}\left\{G^{\prime}(\theta, \tau)-H^{\prime}(2 s-\theta, \tau)-\frac{1}{3}\left[G^{\prime}(\theta, \tau)-H^{\prime}(2 s-\theta, \tau)\right]^{3}\right\} d s,
$$

where we have used a prime to denote differentiation of the function concerned with respect to its first argument. Thus

$$
\begin{aligned}
G_{\theta \tau}(\theta, \tau)=\frac{1}{2} G_{\theta}(\theta, \tau) & -\frac{1}{6}\left[G_{\theta}(\theta, \tau)\right]^{3}-\frac{1}{4}\left\{1-\left[G_{\theta}(\theta, \tau)\right]^{2}\right\} \lim _{T \rightarrow \infty} \frac{1}{T}[H(2 s-\theta, \tau)]_{0}{ }^{T} \\
& -\frac{1}{2} G_{\theta}(\theta, \tau) \lim _{T \rightarrow \infty} \frac{1}{2 T} \int_{-\theta}^{2 T-\theta}\left[H_{\phi}(\phi, \tau)\right]^{2} d \phi+\frac{1}{6} \lim _{T \rightarrow \infty} \frac{1}{2 T} \int_{-\theta}^{2 T-\theta}\left[H_{\phi}(\phi, \tau)\right]^{3} d \phi .
\end{aligned}
$$

If we use the notation

$$
\overline{H_{\theta}{ }^{n}}(\tau)=\lim _{T \rightarrow \infty} \frac{1}{T} \int_{0}^{T}\left[H_{\theta}(\theta, \tau)\right]^{n} d \theta
$$

then this equation can be written in the form

$$
G_{\theta \tau}=\frac{1}{2} G_{\theta}-\frac{1}{6}\left[G_{\theta}\right]^{3}-\frac{1}{2} G_{\theta} \overline{H_{\theta}^{2}}+\frac{1}{6} \overline{H_{\theta}^{3}} .
$$

Introducing the notation $g(\theta, \tau)=G_{\theta}(\theta, \tau)$ and recalling that for fixed end conditions $G=$ $H$, we obtain finally the equation

$$
2 g_{\tau}=g-\frac{1}{3} g^{3}-\overline{g^{2} g}+\overline{\frac{1}{3} g^{3}} .
$$

Since in this case $g(\theta, \tau)$ is $2 l$-periodic in $\theta$,

$$
\overline{g^{n}}(\tau)=\lim _{T \rightarrow \infty} \frac{1}{T} \int_{0}^{T}[g(\theta, \tau)]^{n} d \theta=\frac{1}{2 l} \int_{-l}^{l}[g(\theta, \tau)]^{n} d \theta .
$$

The first moment $\overline{g^{1}}(\tau)$ is identically zero.

Inasmuch as derivatives with respect to $\theta$ are absent, Eq. (4.10) is an ordinary differential equation for $g(\theta, \tau)$. It is to be solved along with the initial values $g(\theta, 0)$ which are known for all $\theta$, for from Eq. (4.2) it follows that

$g(x, 0)=\frac{1}{2}\left[u_{x}(x, 0)+u_{t}(x, 0)\right], g(-x, 0)=\frac{1}{2}\left[u_{x}(x, 0)-u_{t}(x, 0)\right](0 \leq x \leq l)$.

Thus $g(\theta, 0)$ is known for $-l \leq \theta \leq l$, and from the periodicity property can therefore be constructed for all values of $\theta$. 
We shall solve Eq. (4.10) explicitly in the special case when the initial value $u(x, 0)=0$. In that case it follows from Eqs. (4.11) that $g(\theta, 0)$ is an odd function. But from the differential equation (4.10) it is clear that if $g(\theta, 0)$ is odd, then $g(\theta, \tau)$ is also odd for all $\tau>0$. In this case, the odd moments of $g$ are all zero-in particular $\overline{g^{3}}=0$-and Eq. (4.10) reduces to

$$
2 g_{\tau}=\left(1-\overline{g^{2}}\right) g-\frac{1}{3} g^{3} .
$$

Introducing $w(\tau)$ by means of the definition

$$
w(\tau)=\exp \left\{\frac{1}{2} \int_{0}^{T}\left[1-\overline{g^{2}}\left(\tau^{\prime}\right)\right] d \tau^{\prime}\right\}
$$

and writing $g(\theta, \tau)=w(\tau) h(\theta, \tau)$, we reduce Eq. (4.12) to the form

$$
h_{r}(\theta, \tau)=-\frac{1}{6} w(\tau)^{2} h(\theta, \tau)^{3} .
$$

This may be integrated directly, and the solution written in the form

$$
h(\theta, \tau)=h(\theta, 0)\left[1+\frac{1}{3} \phi(\tau) h(\theta, 0)^{2}\right]^{-1 / 2}
$$

where

$$
\phi(\tau)=\int_{0}^{\tau} w(\tau)^{2} d \tau=\int_{0}^{\tau}\left\{\exp \int_{0}^{\tau}\left[1-\overline{g^{2}}\left(\tau^{\prime}\right)\right] d \tau^{\prime}\right\} d \tau
$$

Since $w(0)=1$ and $\phi^{\prime}(\tau)=w(\tau)^{2}$, it follows that

$$
g(\theta, \tau)=g(\theta, 0)\left\{\phi^{\prime}(\tau) /\left[1+\frac{1}{3} \phi(\tau) g(\theta, 0)^{2}\right]\right\}^{1 / 2} .
$$

By substituting the solution (4.14) into the definition (4.13) of $\phi(\tau)$, an equation for $\phi(\tau)$ is obtained which takes the form

$$
\tau-\ln \phi^{\prime}(\tau)=\frac{3}{2 l} \int_{0}^{2 l} \ln \left[1+\frac{1}{3} \phi(\tau) g(\theta, 0)^{2}\right] d \theta .
$$

Having solved this equation for $\phi(\tau)$, we find the function $g(\theta, \tau)$ from Eq. (4.14), and the solution of the wave equation (4.9) has derivatives given by

$u_{x}(x, t)=g(t+x, \tau)+g(t-x, \tau), u_{t}(x, t)=g(t+x, \tau)-g(t-x, \tau)$.

It is of interest to examine the form of the solution for large times. It can be seen from Eq. (4.15) that as $\tau \rightarrow \infty, \phi(\tau) \sim c \exp (\tau / 4)$, where

$$
c^{4}=4 \exp \left\{-\frac{3}{2 l} \int_{0}^{2 l} \ln \left[\frac{1}{3} g(\theta, 0)^{2}\right] d \theta\right\} .
$$

Consequently, from Eq. (4.14), for values of $\theta$ at which $g(\theta, 0) \neq 0$,

$$
g(\theta, \tau) \sim(\sqrt{ } / 2 / 2) \operatorname{sgn}[g(\theta, 0)] .
$$

Thus $g(\theta, \tau)$ tends asymptotically to one or the other of the two constants $\pm \sqrt{ } 3 / 2$, the switch-over from one value to the other occurring at the zeros of the initial value function $g(\theta, 0)$. The regions in which $g(\theta, \tau)$ changes from one value to the other become narrower and narrower as $\tau$ increases, the derivative of $g$ with respect to $\theta$ within those regions becoming larger and larger. For this reason we shall speak of these regions as being shock layers.

We can estimate the width of the shock layers in the following way. Let $g(\theta, 0)$ have zeros at $\theta=\theta_{i}(i=1,2, \cdots)$ and suppose that for $\theta$ near to $\theta_{i}, g(\theta, 0) \simeq k_{i}\left(\theta-\theta_{i}\right)$ where $k_{i}$ 
$=g_{\theta}\left(\theta_{i}, 0\right)$. Then for large $\tau$, the solution $g(\theta, \tau)$ changes from $-\rho(\sqrt{ } 3 / 2)$ to $+\rho(\sqrt{ } 3 / 2)$ as $\theta$ changes from $\theta_{i}-\Delta_{i}$ to $\theta_{i}+\Delta_{i}$, where

$$
\Delta_{i}=k_{i}{ }^{-1}\left[\frac{3 \rho^{2}}{c\left(1-\rho^{2}\right)}\right]^{1 / 2} \exp (-\tau / 8) .
$$

$2\left|\Delta_{i}\right|$ gives the distance in terms of the variable $\theta$ in which a fraction $\rho$ of the jump across the shock layer occurs, and if $\rho$ is given some representative value, say $\rho=0.9$, we can take $\Delta_{i}$ as a measure of the width of the shock layer at $\theta_{i}$. The width decreases exponentially towards zero as $\tau$ increases.

From Eq. (4.2) we obtain the expressions (4.16) for the derivatives of $u$ in the lowestorder approximation. Thus the shock layers in $g(\theta, \tau)$ centered at $\theta=\theta_{i}$ provide two sets of layers in $u_{x}$ and $u_{t}$, centered at the points $x=t-\theta_{i}$ and $x=-t+\theta_{i}$ and moving respectively with velocities of \pm 1 .

Eqs. (4.9) have been used by Myerscough [20] to model the wind-induced oscillations of overhead powerlines, and the results of the present section allow further conclusions to be drawn regarding the behavior of such oscillations. To the extent that the model is applicable, any oscillation $u$ caused by an initial impulse $\left(u_{t}(x, 0) \neq 0, u(x, 0)=0\right)$ asymptotically tends to the superposition of two saw-tooth waves, one travelling in each direction. The slopes of the saw-teeth are $\pm \sqrt{ } 3 / 2$, so that the maximum amplitude of oscillation is asymptotically $\sqrt{ } 3 l / 2$. This compares with the result $4 l / \sqrt{ } 3 \pi$ obtained from simple energy considerations [20], these two values being reasonably close to one another. Some of the numerical solutions presented by Myerscough are qualitatively consistent with the saw-tooth type of asymptotic solution.

4.4 Free end conditions. In the case when $u(x, t)$ satisfies Eq. (4.1) in the region $0<x$ $<l$ together with the boundary conditions $u_{x}(0, t)=u_{x}(l, t)=0$, the method of even extension may be used. The function $u(x, t)$ is extended to all values of $x$ according to the prescriptions $u(-x, t)=u(x, t), u(x+2 l, t)=u(x, t)$, so that $u(x, t)$ is even about both $x=$ 0 and $x=l$. Eq. (4.1) continues to be satisfied for all $x$ provided that the function $E$ is extended according to the following definitions:

$$
\begin{aligned}
E\left(-x, t, u,-u_{x}, u_{t}, \cdots, \epsilon\right) & =E\left(x, t, u, u_{x}, u_{t}, \cdots, \epsilon\right) \\
E\left(x+2 l, t, u, u_{x}, u_{t}, \cdots, \epsilon\right) & =E\left(x, t, u, u_{x}, u_{t}, \cdots, \epsilon\right) .
\end{aligned}
$$

With $u_{0}$ given by Eq. (4.2), the requirement that it be even about $x=0$ and $x=l$ is met by assuming that $G(\theta, \tau)=-H(\theta, \tau)$ and that $G$ and $H$ are periodic in their first arguments with period 2l. These assumptions can be seen to be compatible with Eqs. (4.4) provided that $E$ satisfies the conditions (4.17). The solution in lowest order thus takes the form

$$
\begin{gathered}
u=G(t+x, \tau)+G(t-x, \tau), \\
u_{x}=g(t+x, \tau)-g(t-x, \tau), u_{t}=g(t+x, \tau)+g(t-x, \tau)
\end{gathered}
$$

where $g(\theta, \tau)=G_{\theta}(\theta, \tau)$.

Consider the example in Eq. (4.5). The extended function $E$ from Eqs. (4.17) is given by

$$
E=2(-1)^{p} u_{x} u_{x x}+k u_{x x t}, p l<x<(p+1) l .
$$

By substituting this into Eq. (4.4), it can be shown that after taking the limit $T \rightarrow \infty$, the nonlinear term in $E$ makes no contribution to $G_{\theta^{\tau}}$. Eq. (4.4) reduces simply to the conduction equation,

$$
g_{\tau}(\theta, \tau)=\frac{1}{2} k g_{\theta \theta}(\theta, \tau)
$$


This feature of Eq. (4.5), that the nonlinear terms do not affect the lowest-order solution when the boundary conditions are of free-end type, has been pointed out previously by Mortell and Varley [21] (see also [10]).

The form $E=u_{t}-\frac{1}{3} u_{t}{ }^{3}$ pertaining to Eq. (4.9) automatically satisfies the conditions (4.17), so that the solution continues to be given by Eqs. (4.10), (4.18). In this case, however, $g(\theta, 0)$ is an odd function when $u_{t}(x, 0)=0$, so that the solution (4.14), (4.15) applies when the initial disturbance consists of a non-zero displacement but zero velocity.

\section{REFERENCES}

[1] N. N. Krylov and N. N. Bogoliubov, Introduction to nonlinear mechanics, University of Princeton Press, 1947

[2] N. N. Bogoliubov and Yu. A. Mitropolskii, Asymptotic methods in the theory of nonlinear oscillations, Gordon and Breach, 1961

[3] D. J. Benney, Nonlinear gravity-wave interactions, J. Fluid Mech. 14, 577-584 (1962)

[4] L. F. McGoldrick, Resonant interactions among capillary-gravity waves, J. Fluid Mech. 21, 305-331 (1965)

[5] D. Montgomery and D. A. Tidman, Secular and non-secular behaviour for the cold plasma equations, Phys. Fluids 7, 242-249 (1964)

[6] D. Montgomery, Generalized perturbation expansion for the Klein-Gordon equation with small nonlinearity, J. Math. and Phys. 5, 1788-1795 (1964)

[7] Yu. A. Mitropolskii and B. J. Moseenkov, Lectures on the application of asymptotic methods to the solution of partial differential equations, Academy of Sciences of Ukr. SSR, Kiev, 1968

[8] G. N. Bojadziev and R. W. Lardner, Monofrequent oscillations in mechanical systems governed by secondorder hyperbolic differential equations with small nonlinearities, Int. J. Nonlinear Mech. 8, 289-309 (1973)

[9] G. N. Bojadziev and R. W. Lardner, Second-order hyperbolic equations with small nonlinearities in the case of internal resonance, Int. J. Nonlinear Mech. 9, 397-407 (1974)

[10] R. W. Lardner, The formation of shock waves in KBM solutions of hyperbolic partial differential equations, J. Sound Vibr. 39, 489-502 (1975)

[11] R. W. Lardner, The development of plane shock-waves in nonlinear viscoelastic media, Proc. Roy. Soc. A 347, 329-344 (1976)

[12] J. D. Cole and J. Kevorkian, Uniformly valid approximations for certain nonlinear differential equations, in Proc. Int. Symp. on Nonlinear Diff. Eqs. and Nonlinear Mechs., ed. J. P. LaSalle and S. Lefschetz, Academic Press, 1963, pp. 113-120

[13] J. A. Morrison, Comparison of the modified method of averaging and the two variable expansion procedure, SIAM Review 8, 66-85 (1966)

[14] A. H. Nayfeh, Perturbation methods, chap. VI, John Wiley, 1973

[15] J. B. Keller and S. Kogelman, Asymptotic solutions of initial value problems for nonlinear partial differential equations, SIAM J. Appl. Math. 18, 748-758 (1970)

[16] D. T. Davy and W. E. Ames, An asymptotic solution of an initial value problem for a nonlinear viscoelastic rod, Int. J. Nonlinear Mech. 8, 59-71 (1973)

[17] A. H. Nayfeh, Finite amplitude longitudinal waves in non-uniform bars, J. Sound Vibr. 42, 357-361 (1975)

[18] S. C. Chikwendu and J. Kevorkian, A perturbation method for hyperbolic equations with small nonlinearities, SIAM J. Appl. Math. 22, 235-258 (1972)

[19] J. P. Fink, W. S. Hall and A. R. Hausrath, Convergent two-time method for periodic differential equations, J. Diff. Eqs. 15, 459-498 (1974)

[20] W. Eckhaus, New approach to the asymptotic theory of nonlinear oscillations and wave propagation, J. Math. Anal. Applic. 49, 575-611 (1975)

[21] C. J. Myerscough, A simple model of the growth of wind-induced oscillations in overhead lines, J. Sound Vibr. 28, 699-713 (1973); 39, 503-517 (1975)

[22] M. P. Mortell and E. Varley, Finite amplitude waves in bounded media: nonlinear free vibration of an elastic panel, Proc. Roy. Soc. A 318, 169-196 (1970)

[23] M. P. Mortell and B. R. Seymour, Pulse propagation in a nonlinear viscoelastic rod of finite length, SIAM J. Appl. Math. 22, 209-223 (1972)

[24] J. C. Arya and R. W. Lardner, Plane shock waves in viscoelastic media displaying cubic elasticity, SFU preprint, 1977 